\title{
The grand challenges in cardiovascular drug discovery and development
}

\author{
Eliot H. Oh/stein ${ }^{1,2 *}$ \\ 1 AltheRx Pharmaceuticals, Philadelphia, PA, USA \\ School of Medicine, Drexel University, Philadelphia, PA, USA \\ *Correspondence: eliot@altherx.com
}

Despite the major advancements in cardiovascular medicine over the past 50 years, heart disease and stroke remain the number one public health concern and primary causes of death today. The development of effective medicines that control blood pressure and cholesterol has significantly cut deaths from heart disease. According to a 2008 report, death rates for cardiovascular disease fell a remarkable $26.4 \%$ between 1999 and 2005. Nonetheless, $45 \%$ of Americans still have hypertension, high cholesterol or diabetes. According to a 2006 American Heart Association study cardiovascular disease accounted for over a third of all deaths in the United States. On average, every 40 seconds someone in the United States has a stroke. Heart disease and stroke are becoming a worldwide pandemic as developing nations are witnessing the health burden. In developing nations approximately 14 million individuals died of cardiovascular disease in 1990 , and deaths are projected to rise to 25 million by 2020 .

What are the Grand Challenges that must be addressed to confront the obstacles for the creation of new medicines that will treat patients with cardiovascular disease? These Grand Challenges will be broken it into three broad categories:

1) Innovation;

2) Translating basic research into clinical reality;

3) Commitment

\section{INNOVATION}

Breakthroughs in elucidating the basic molecular pharmacology in cardiac/vascular function, thrombosis/coagulation and lipid metabolism, has lead to significant advancements in the current treatment paradigm for patients with heart disease. Innovation in these areas of research has lead to remarkable discoveries and significantly reduced the mortality and morbidity from cardiovascular diseases. Notwithstanding, more work and a relentless pursuit of the basic science in cardiovascular biology is still needed.

Innovation is evident in the field of regenerative medicine. Regenerative medicine is an emerging interdisciplinary field of scientific research and clinical applications that will impact significantly on the treatment of heart disease and diabetes. Research focused on the repair, replacement, or regeneration of cardiac, vascular, or pancreatic tissues to restore function resulting from disease holds much promise. Cardiac regenerative medicine may use a combination of several technological approaches including, but not limited to, the use of stem cells, soluble molecules, genetic and tissue engineering, and advanced cell therapy. Promising clinical research is already available for the use of autologous bone marrow stem cells for the regeneration of ischemic heart muscle after myocardial infarction and cardiomyopathies. In addition to saving lives, such therapies will markedly reduce the cost of treating patients with heart failure. Moreover, regenerative medicine therapies are relevant in the consideration of new therapeutic approaches for diabetes. Bone marrow stem cell transplantation or microencapsulated pancreatic islet cells using novel biomaterials could potentially provides a permanent solution and prevent the burden of diabetes.

Innovation is not solely found with small molecule compounds. Biopharmaceuticals have the opportunity to tackle previously intractable targets. The concept of vaccines to treat cardiovascular disease is now feasible. Moreover, medical device approaches such as drug eluting stents, implantable cardiac defibrillators, pacemakers, insulin pumps, and other devices will certainly have a place for new innovative approaches to treat cardiovascular disease and will continue to make an impact.
Innovation in the field of genetics and personalized medicine will be integrated into the discovery and development of new cardiovascular therapeutics. New genetic knowledge opens up the possibility of developing "targeted" therapies for people with specific gene sequences, and it will help physicians choose from amongst existing medicines treatment that best meets an individuals genetic, lifestyle, and environmental differences. In addition, research is evolving for the development of genetic tests that tells if patients are susceptible to certain types of disease.

\section{TRANSLATING BASIC RESEARCH INTO CLINICAL REALITY}

Making new drugs is hard. Sometimes an obvious therapeutic target with excellent preclinical data does not translate into a safe and effective medicine. The recent example of the failure of the CETP inhibitor Torcetrapib illustrates this problem. It was widely accepted that increasing HDL produces a beneficial therapeutic effect and decrease atherosclerosis. People were stunned when Torcetrapib failed to translate the biochemistry and pharmacology into a clinical benefit. Although the hypothesis that inhibition of CETP to increase HDL is still viable and compounds are still being developed, it cast doubt on the sole reliance of established biomarkers for the efficacy of potential new drugs.

The vasopeptidase inhibitor Omapatrilat is another example of how high-quality cardiovascular drug discovery and development failed to produce an approvable medicine. Omapatrilat demonstrated excellent antihypertensive efficacy in hypertensive patients; however, there was a slightly higher incidence of severe angioedema than the standard of care ACE inhibitor. Omapatrilat was not approved because of these safety concerns. Transformational achievements from the past two decades for safe and effective antihypertensive 
medicines raised the bar in terms of safety. New drugs for most cardiovascular diseases will need to be "squeaky-clean" for patient safety. Thus the hurdles for regulatory approval have been raised.

In today's environment of less funding, less validated targets, greater regulatory hurdles and larger clinical studies, there will be less risk-taking for companies to develop new cardiovascular therapeutics. This is a "Grand Challenge" but we must ask how can this trend be reversed? In order to improve the successful translation of research the emphasis in early development should be on understanding the molecular underpinnings of disease and developing biomarkers for therapeutic relevance. The emerging technologies of genomics, proteomics, and metabolomics combined with physiological profiling and imaging modalities will provide valuable insight. Differential gene and protein expression in disease or following drug treatment will instill valuable insight into the disease process.

Research shows that cardiovascular disease has a signature of molecular biomarkers. Biomarker signatures may dictate how a patient might respond to treatment. This is a powerful tool that will detect certain diseases at their earliest stages before the onset of symptoms and when they are most treatable and preventable. Biomarkers represent the future of cardiovascular medicine, in which disease diagnosis, treatment, monitoring and prevention will be guided by a continual readout of a patient's molecular composition. The employment of novel biomarker strategies in clinical trials should serve to mitigate clinical development risk.

Increasingly, the administration of new medicines for cardiovascular disease will be guided by predictive evidence from genetic and other molecular tests. As stated above, the expectation of "personalized medicine" is that these tests will reveal whether an individual is likely to respond well to a drug, or avoid toxic side effects. A targeted approach to treatment will ensure that each patient receives the right medicine at the right time. Since molecular diagnostic tests reveals a patient's susceptibility to disease, they also guide preventive treatment before symptoms arise.

A common saying in the pharmaceutical industry is to "fail early." Toxicity identified during clinical trials is a major cause of attrition during drug development. Quick identification of those drugs that will not have successful target validation or those drugs that will not have sufficient safety is a critical element of modern drug discovery strategies. To make this process more efficient, and to prevent unexpected safety issues late in the development process, scientists are seeking new methods to predict toxic effects early in the discovery process. Alternatives to traditional animal toxicology testing are being integrated into the early discovery and development process. Predictive toxicology is now one of the first steps in early drug discovery. In silico and in vitro technological methods are being used to predict in vivo results, thus a company can cost effectively develop drugs that will increase the probability of making it to the market.

\section{COMMITMENT}

Drug discovery and development in cardiovascular disease has previously been sponsored by large investments by the pharmaceutical industry. We are witnessing a change in this paradigm. As a consequence of the successes in cardiovascular medicine over the past decade many pharmaceutical companies are shifting their focus and resources into different therapeutic areas. The incentive to develop cardiovascular agents has been reduced by increasing clinical development costs coupled with an augmented risk of failure due to the unprecedented nature of the promising drug targets and an increasingly challenging regulatory environment. In addition, the global recession and fundamental concerns about the lack of $\mathrm{R} \& \mathrm{D}$ productivity have resulted in substantial industry layoffs over the past 2 years. Furthermore, academic granting agencies have made a similar shift in funding into different therapeutic areas. Consequently there are less scientists employed doing cardiovasculardirected research today.

We are reaching a peak in the development pipeline of drugs for cardiovascular disease. Investment from the previous decade resulted in new potential medicines to treat cardiovascular disease; however, future investment in cardiovascular disease is uncertain. Where will new innovation in cardiovascular medicine come from? New medicines for heart disease are still needed and most of the basic science and discovery will occur in academic laboratories. Academic laboratories and the biotech industry need to take on more responsibility for validating new targets and translating the science into commercially available medicines. In addition, the pharmaceutical industry will be externalizing many discovery activities in order to access new discoveries and exploit the power of early stage innovative technologies.

Academia and the biotech industry need to create new partnerships with the pharmaceutical industry for the resources and expertise that are essential for the clinical trials and the overall drug development process. The science is too complex, and the risks are too high. Companies will increasingly build alliances to bring novel therapeutic agents to the market. New models of partnerships between academia and the biotech industry, and the pharmaceutical industry will be critical to advance the new discoveries into new cardiovascular medicines.

\section{CONCLUSIONS}

The challenge in the advancement of new cardiovascular therapeutics goes beyond scientific breakthroughs and innovation. Issues involving patient access to medical care, smoking, physical activity, strategies to combat obesity, malnutrition, risk factor modification, regulatory, and public health policies that enable economic development of new cardiovascular medicines must be addressed before the benefits of new scientific discoveries will be fully realized. Although the burden of cardiovascular disease is already emerging in developing countries, there exists a window of opportunity to prevent the pandemic from reaching its full potential magnitude. If the challenges of Innovation, Translation, and Commitment are addressed, we may be successful in preventing cardiovascular disease from becoming the number one global health burden.

Received: 02 September 2010; accepted:08 September 2010; published online: 23 September 2010.

Citation: Ohlstein EH (2010) The grand challenges in cardiovascular drug discovery and development. Front. Pharmacol. 1:125. doi: 10.3389/fphar.2010.00125

This article was submitted to Frontiers in Cardiovascular and Smooth Muscle Pharmacology, a specialty of Frontiers in Pharmacology.

Copyright (C) 2010 Ohlstein. This is an open-access article subject to an exclusive license agreement between the authors and the Frontiers Research Foundation, which permits unrestricted use, distribution, and reproduction in any medium, provided the original authors and source are credited. 